\title{
APPROXIMATION OF ILL-POSED BOUSSINESQ EQUATIONS
}

\author{
DIOGO A. GOMES AND CLAUDIA VALLS
}

\begin{abstract}
In this paper we compare the dynamics on the center manifold of the solutions of a ill posed Boussinesq equation with a well posed version. We show that the dynamics in the center manifold of the ill posed equation tracks the dynamics of the well posed equation. Our results give partial justification to the long wave perturbation theory.
\end{abstract}

\section{INTRODUCTION}

Boussinesq [2] developed a perturbation theory for surface motions in a long wave regime and developed models of the motion of the free surface that are very good approximations of the classical problem. Boussinesq-type equations also arise in several other physical applications such as vibrations in a nonlinear string, and ion sound waves in a plasma, as well as being a model to study nonlinear partial differential equations. Boussinesq's perturbation theory yields simplified equations that give realistic results in the appropriate regimes. One of these equations is

$$
(1-\alpha \Delta) u_{t t}=u_{x x}+\varepsilon\left(u^{2}\right)_{x x}, \quad \alpha>0,
$$

where $\alpha, \varepsilon>0$ are parameters which are proportional, respectively, to the square of the inverse of wave lenght and wave amplitude. Therefore, the parameter $\epsilon$ measures the $L^{2}$ norm of the solution of the original water wave problem. We take $x \in[0,1]$, and we impose periodic boundary conditions $u(t, 0)=u(t, 1), 0 \leq t<\infty$, and zero mean $\int_{0}^{1} u d x=0$. Periodic boundary conditions, besides being mathematically convenient, are relevant for the study of water waves in a large circular channel in which curvature effects are neglected. In the long wave regime the perturbation theory yields the well known Boussinesq equation, given by

$$
u_{t t}=u_{x x}+\alpha u_{x x x x}+\varepsilon\left(u^{2}\right)_{x x}, \quad \alpha>0 .
$$

Although equation (2) may look simpler than equation (1), it turns out to be ill-posed thus causing a substantial additional complication.

The main objective of this paper is is to compare the flow on the centre manifold of the illposed Boussinesq equation (2), with the flow of the wellposed Boussinesq equation (1) in order to give some justification to this

D. G. was partially supported by FCT/POCTI/FEDER; C. V. was partially supported by the FCT grant SFRH/BPD/14404/2003. 
long wave approximation theory. The main question we want to address is whether the center manifold solutions to (2) track the dynamics of a submanifold of (1). To do so, we use techniques of center manifold theory in [6], and show that nontrivial solutions of (2) in the center manifold approximate solutions of (1) for long times. See Theorem 6 below for a precise statement.

We remark that the approximation property only holds for suitable initial data of (2). In fact, even the linear part of (2) exhibits blow up for general initial conditions in arbitrarily small time. It is believed that the nonlinear equation shares these features. This is in contrast with equation (1) which is known to be well-posed (see Theorem 1). However, we believe that the blow-up mechanism for (2) does not reflect the physics of the problem but instead that the finite-dimensional approximations of the center manifold that we construct should contain all the relevant physical information. We should also note that this is not a long time existence and approximation result, meaning approximation time going to $\infty$ as the parameters vanish, as discussed, for instance, [1].

The plan of the paper is as follows. In Section 2 we briefly review the existence of solutions of (1) by energy methods and the center manifold theory for (2). In Section 3 we construct finite-dimensional approximations to (2) and (1), and show that these are close to each other. In section 4 we prove that the approximations stay close to the solutions of the respective equations. This indicates that (2) must be considered a suitable approximation of (1) for long times.

We would like to thank Luis Barreira for many useful comments and suggestions he made on a previous version of this paper.

\section{Preliminaries}

Consider the Sobolev space

$$
H^{k}=\left\{u \in L_{\mathrm{loc}}^{1}[0,1]: D^{\alpha} u \in L^{2}[0,1] \text { for all } 0 \leq|\alpha| \leq k\right\},
$$

where $D^{\alpha} u$ is the weak derivative of $u$. For functions with zero mean, the norm is given by

$$
\|u\|_{H^{k}}=\sum_{0<|\alpha| \leq k} \int_{0}^{1}\left|D^{\alpha} u\right|^{2} d x .
$$

By Poincaré's inequality,

$$
\|u\|_{L^{2}[0,1]} \leq C\|u\|_{H^{k}}
$$

for some constant $C>0$. The space of bounded functions in $[a, b] \subset \mathbb{R}$ with values in $H^{k}$ is denoted by $L^{\infty}\left([a, b], H^{k}\right)$, and has the norm

$$
\|u\|_{L^{\infty}\left([a, b], H^{k}\right)}=\operatorname{ess~sup}_{t \in[a, b]}\|u(\cdot, t)\|_{H^{k}} .
$$

These have a natural identification with the functions in $[a, b] \times[0,1]$ that for almost every $t \in[a, b]$ are in $H^{k}$. For simplicity, we shall write $u \in$ $L^{\infty}\left([a, b], H^{k} \times H^{j}\right)$ for $\left(u, u_{t}\right) \in L^{\infty}\left([a, b], H^{k} \times H^{j}\right)$. 
We first use energy methods to establish the well-posedness of (1).

Theorem 1. If $\left(\left.u\right|_{t=0},\left.u_{t}\right|_{t=0}\right) \in H^{m} \times H^{m}, m \geq 1$, then there exists $T_{*}>0$ and a solution $u$ of $(1)$ such that $u \in L^{\infty}\left(\left[0, T_{*}\right], H^{m} \times H^{m}\right)$. Furthermore, $T_{*}$ blows up as $\frac{\epsilon}{\alpha^{1 / 2}} \rightarrow 0$.

Proof. We first establish a priori energy estimates. The theorem then follows from standard techniques (see [7], for example).

We assume that a solution $u$ exists and is sufficiently differentiable. Applying $D^{m-1}$ to (1) and multiplying by $D^{m-1} u_{t}$, we have

$$
\begin{aligned}
& \left(D^{m-1} u_{t t}\right)\left(D^{m-1} u_{t}\right)-\alpha\left(D^{m+1} u_{t t}\right)\left(D^{m-1} u_{t}\right)= \\
& =\left(D^{m-1} u_{t}\right)\left(D^{m-1} u_{x x}\right)+\varepsilon\left(D^{m-1}\left(u^{2}\right)_{x x}\right)\left(D^{m-1} u_{t}\right) .
\end{aligned}
$$

Let now

$$
E=\int_{0}^{1}\left[\left(D^{m-1} u_{t}\right)^{2}+\alpha\left(D^{m} u_{t}\right)^{2}+\left(D^{m} u\right)^{2}\right] d x .
$$

Integrating by parts, it follows from (3) that

$$
\frac{1}{2} \frac{d}{d t} E=-\varepsilon \int_{0}^{1}\left(D^{m} u_{t}\right)\left(D^{m}\left(u^{2}\right)\right) d x
$$

Using the Cauchy-Schwartz inequality in (4), we have

$$
\frac{d}{d t} E \leq 2 \varepsilon\left(\int_{0}^{1}\left(D^{m} u_{t}\right)^{2} d x\right)^{1 / 2}\left(\int_{0}^{1}\left(D^{m} u^{2}\right)^{2} d x\right)^{1 / 2} .
$$

Since

$$
\int_{0}^{1}\left(D^{m} u_{t}\right)^{2} d x \leq \frac{E}{\alpha} \quad \text { and } \quad \int_{0}^{1}\left(D^{m} u^{2}\right)^{2} d x \leq\left\|u^{2}\right\|_{H^{m}}^{2}
$$

we conclude that

$$
\frac{d}{d t} E \leq 2 \varepsilon \frac{E^{1 / 2}}{\alpha^{1 / 2}}\left\|u^{2}\right\|_{H^{m}} .
$$

There exists a constant $C>0$, such that since $H^{m}, m>1 / 2$ is an algebra, $\left\|u^{2}\right\|_{H^{m}} \leq C\|u\|_{H^{m}}^{2}$, and since $\int_{0}^{1} u d x=0,\|u\|_{H^{m}}^{2} \leq C E$, by the Poincaré inequality. Thus,

$$
\frac{d}{d t} E \leq C^{\prime} \frac{\varepsilon}{\alpha^{1 / 2}} E^{3 / 2}
$$

for some constant $C^{\prime}>0$. This inequality implies local existence for small data. The maximum time for the existence of the solution, $T_{*}$, is obtained by considering an equality in (5) and finding the blow up time $T_{*}$ at which $E$ goes to infinity. Denoting $E_{0}=E(t=0)$, we have

$$
T_{*}=2 \alpha^{1 / 2} /\left(C^{\prime} \varepsilon E_{0}^{1 / 2}\right) \quad \text { and } \quad u \in L^{\infty}\left(\left[0, T_{*}\right], H^{m} \times H^{m}\right) .
$$

This completes the proof. 
We note that $T_{*} \rightarrow 0$ as $\alpha \rightarrow 0$, unless $\varepsilon^{2} \sim \alpha$ or $E \sim O(\alpha)$.

We now apply the center manifold theory to construct a finite-dimensional manifold invariant under (2). The next theorem was proved in an unpublished paper [4] using a new version of the center manifold theorem. However, as pointed out by one of the referees, the same result could be obtained using Mielke's center manifold theorem from [6], chapter 2. Therefore, for the convenience of the reader, we present a sketch of the proof using this result.

Theorem 2. For each $r>1$, there is a $C^{r}$ finite-dimensional manifold $W \subset H^{m} \times H^{m-2}$, for $m \geq 2$, containing 0 which is locally invariant under (2) in some neighborhood of the origin, and such that $T_{0} W$ is spanned by the eigenvectors of the linear part of (2) with purely imaginary eigenvalues. Furthermore, the solutions in this manifold are locally well-posed in time up to $t=T_{\sharp}$ which blows up as $\epsilon \rightarrow 0$.

Proof. This theorem is a straightforward application of theorem 2.1 from [6]. We just sketch the main points.

One can easily write (2) as a system

$$
\left\{\begin{array}{l}
u_{t}=v \\
v_{t}=u_{x x}+\alpha u_{x x x x}+\epsilon\left(u^{2}\right)_{x x} .
\end{array}\right.
$$

The eigenvalues of the linearized part $K: H^{m+2} \times H^{m} \rightarrow H^{m} \times H^{m-2}$,

$$
K(u, v)=\left[\begin{array}{c}
v \\
u_{x x}+\alpha u_{x x x x}
\end{array}\right],
$$

are given by

$$
\left\{\lambda_{k}(\alpha)\right\}_{k \geq 1}=\left\{ \pm 2 \pi k \sqrt{\alpha 4 \pi^{2} k^{2}-1}\right\}_{k \geq 1},
$$

only finitely many lie on the imaginary axis, and there are no zero eigenvalues (since we have the zero mean condition). Thus $K$ can be written as $K_{1}+K_{2}$ in which, $K_{1}$ corresponds to the projection in the imaginary eigenspace and so $e^{t K_{1}}$ generates a strongly continuous group; $K_{2}$ satisfies the resolvent estimate

$$
\left\|\left(K_{2}-i \xi\right)^{-1}\right\| \leq \frac{C}{1+|\xi|}, \quad \xi \in \mathbb{R}
$$

for some $C>0$. Furthermore, the nonlinearity $\left[0,\left(u^{2}\right)_{x x}\right]$ is also $C^{k}$ from $H^{m+2} \times H^{m}$ to $H^{m} \times H^{m-2}$. The space $T_{0} W$ is spanned by the eigenvectors corresponding to the eigenvalues $\left\{\lambda_{k}(\alpha)\right\}_{k<\bar{k}}$, with $\bar{k}=\left[1 / \sqrt{4 \pi^{2} \alpha}\right]$, where [.] denotes the integer part.

To check that $T_{\sharp}$ blows up as $\epsilon \rightarrow 0$ it suffices to use a scaling argument. In fact, $\hat{u}=\epsilon u$ solves (2) with $\epsilon=1$. Therefore, since $\hat{u} \rightarrow 0$, its existence time blows up as $u=0$ is a equilibrium point. 


\section{Finite Dimensional apProximations}

Our approach consists in constructing approximate solutions to (2) and (1) using only a finite number of Fourier modes. To this end, we assume that the parameter $\varepsilon$ is sufficiently small and write an approximate solution $\tilde{u}$ of equation (2) as the finite sum

$$
\tilde{u}=v_{0}+\varepsilon v_{1}+\varepsilon^{2} v_{2}+\cdots+\varepsilon^{M} v_{M},
$$

with $v_{0} \neq 0, v_{1}=\cdots=v_{M}=0$ at $t=0$. Each function $v_{i}, 0 \leq i \leq M$ satisfies a linear equation. These equations are

$$
\left(v_{0}\right)_{t t}=\left(v_{0}\right)_{x x}+\alpha\left(v_{0}\right)_{x x x x},
$$

and, for $1 \leq i \leq M$,

$$
\left(v_{i}\right)_{t t}=\left(v_{i}\right)_{x x}+\alpha\left(v_{i}\right)_{x x x x}+N_{i}\left(v_{0}, \ldots, v_{i-1}\right),
$$

where

$$
N_{i}\left(v_{0}, \ldots, v_{i-1}\right)=\left(\sum_{j=0}^{i-1} v_{j} v_{i-j-1}\right)_{x x} .
$$

Similarly, for equation (1) we consider the approximation

$$
\bar{u}=w_{0}+\varepsilon w_{1}+\varepsilon^{2} w_{2}+\cdots+\varepsilon^{M} w_{M},
$$

with $w_{0} \neq 0, w_{1}=\cdots=w_{M}=0$ at $t=0$. We obtain the linear equations

$$
(1-\alpha \Delta)\left(w_{0}\right)_{t t}=\left(w_{0}\right)_{x x}
$$

and, for $1 \leq i \leq M$,

$$
(1-\alpha \Delta)\left(w_{i}\right)_{t t}=\left(w_{i}\right)_{x x}+N_{i}\left(w_{0}, \ldots, w_{i-1}\right)
$$

with the functions $N_{i}$ as in (8).

It is important to note that if $v_{0}, w_{0}$ have only the first $N$ Fourier modes different from zero, then, for $M \geq 1, v_{M}$ and $w_{M}$ have at most $(M+1) N$ Fourier modes different from zero. These approximations can be determined by solving a system of ordinary differential equations. Note that these equations are approximations to the dynamics for fixed time T. Another approach could be to use a normal form and truncate the Hamiltonian system. This would have the advantage of preserving the Hamiltonian structure, however there could be ressonances which would make the analysis more complex. Since they are linear ordinary differential equations, the solutions exist for all times and one has bounds of the type

$$
\|v\|,\|w\| \leq c e^{c\left(N^{2}+\alpha N^{4}\right) t} .
$$

Thus, these approximations, and, in particular, the ones for equation (2), are well-posed for such initial conditions and bounded in any Sobolev space. Note that these estimates are far from optimal as we are not using the 
structure of the equations. For instance, the first order approximation $v_{0}$ satisfies

$$
\frac{d}{d t} \int\left(v_{0}\right)_{t}^{2}+\left(v_{0}\right)_{x}^{2}-\alpha\left(v_{0}\right)_{x x}^{2}=0
$$

which for $\alpha$ small enough controls the norm of $v_{0}$. The same type of remark could be made in the next lemma. However, the critical estimates are the existence time in the center manifold, and lemmas 4 and 5. Therefore, any possible improvements in the results of this section would not change the main result, unless additional hypothesis were added, see, however, the remarks after the statements of lemmas 4 and 5 .

We assume that the initial conditions for both approximations coincide, that is $v_{i}(x, 0)=w_{i}(x, 0)$, for $0 \leq i \leq M$. The following lemma ensures the proximity between $v_{i}$ and $w_{i}$ for each $0 \leq i \leq M$.

Lemma 3. Suppose that $v_{0}, w_{0}$ have only the first $N$ Fourier modes different from zero. Let $m \geq 0$. For $\alpha$ sufficiently small and $T \ll 1 / \alpha$, there exists a constant $K_{i} \equiv K_{i}(N)$ such that, for $0 \leq i \leq M$,

$$
\sup _{0 \leq t \leq T}\left\|v_{i}-w_{i}\right\|_{H^{m} \times H^{m}} \leq K_{i} \alpha .
$$

Proof. We proceed by induction on $i$. For $i=0, v_{0}$ and $w_{0}$ satisfy

$$
\left(v_{0}\right)_{t t}=\left(v_{0}\right)_{x x}+\alpha\left(v_{0}\right)_{x x x x} \text { and }(1-\alpha \Delta)\left(w_{0}\right)_{t t}=\left(w_{0}\right)_{x x} .
$$

These can respectively be written as

$$
\frac{d}{d t} z_{0}=A z_{0}, \quad z_{0}=\left(\begin{array}{c}
v_{0} \\
\left(v_{0}\right)_{t}
\end{array}\right), \quad A=\left(\begin{array}{cc}
0 & 1 \\
\Delta+\alpha \Delta^{2} & 0
\end{array}\right),
$$

and

$$
\frac{d}{d t} y_{0}=B y_{0}, \quad y_{0}=\left(\begin{array}{c}
w_{0} \\
\left(w_{0}\right)_{t}
\end{array}\right), \quad B=\left(\begin{array}{cc}
0 & 1 \\
\frac{\Delta}{1-\alpha \Delta} & 0
\end{array}\right) .
$$

Note that

$$
\frac{d}{d t}\left(z_{0}-y_{0}\right)=A\left(z_{0}-y_{0}\right)+(A-B) y_{0}
$$

We first estimate the term $A\left(z_{0}-y_{0}\right)$. Since $z_{0}$ and $y_{0}$ have only the first $N$ Fourier modes different from zero,

$$
\begin{aligned}
\left\|A\left(z_{0}-y_{0}\right)\right\|_{H^{m} \times H^{m}} & \leq\left\|\left(v_{0}\right)_{t}-\left(w_{0}\right)_{t}\right\|_{H^{m}}+N^{2}\left(1+\alpha N^{2}\right)\left\|v_{0}-w_{0}\right\|_{H^{m}} \\
& \leq N^{2}\left(1+\alpha N^{2}\right)\left\|z_{0}-y_{0}\right\|_{H^{m} \times H^{m}},
\end{aligned}
$$

with the norm

$$
\left\|\left(w_{1}, w_{2}\right)\right\|_{H^{m} \times H^{m}}=\left\|w_{1}\right\|_{H^{m}}+\left\|w_{2}\right\|_{H^{m}} .
$$

To handle the term $(A-B) y_{0}$, note that

$$
A-B=\left(\begin{array}{cc}
0 & 0 \\
\Delta+\alpha \Delta^{2}-\frac{\Delta}{1-\alpha \Delta} & 0
\end{array}\right) .
$$


From the Taylor expansion, which is valid since the function we are considering have only a small number of Fourier coefficients and $\Delta$ is then a bounded operator,

$$
\frac{\Delta}{1-\alpha \Delta}=\Delta+\alpha \Delta^{2}+O\left(\alpha^{2} \Delta^{3}\right)
$$

we obtain

$$
A-B=\left(\begin{array}{cc}
0 & 0 \\
O\left(\alpha^{2} \Delta^{3}\right) & 0
\end{array}\right) .
$$

Consequently, taking $\alpha$ sufficiently small depending on $N$, we conclude that there exists a positive constant $C(N)$ such that

$$
\left\|(A-B) y_{0}\right\|_{H^{m} \times H^{m}} \leq C(N) \alpha^{2}\left\|y_{0}\right\|_{H^{m}} .
$$

Therefore,

$$
\frac{d}{d t}\left\|z_{0}-y_{0}\right\|_{H^{m} \times H^{m}} \leq N^{2}\left(1+\alpha N^{2}\right)\left\|z_{0}-y_{0}\right\|_{H^{m} \times H^{m}}+C(N) \alpha^{2}\left\|y_{0}\right\|_{H^{m}} .
$$

By Gronwall's inequality, for $t \geq 0$,

$$
\left\|z_{0}-y_{0}\right\|_{H^{m} \times H^{m}} \leq C_{1}(N) \alpha^{2}\left\|y_{0}\right\|_{L^{\infty}\left([0, t], H^{m}\right)} e^{N^{2}\left(1+\alpha N^{2}\right) t} .
$$

Hence, taking $T \ll 1 / \alpha$,

$$
\sup _{0 \leq t \leq T}\left\|z_{0}-y_{0}\right\|_{H^{m} \times H^{m}} \leq C_{2}(N) \alpha^{2}\left\|y_{0}\right\|_{L^{\infty}\left([0, T], H^{m}\right)} .
$$

Note that $y_{0}$, since it only has a finite number of degrees of freedom, its Fourier modes, is the solution of a finite-dimensional linear ordinary differential equation with constant coefficients. Hence, it is bounded for all times, and so $\left\|y_{0}\right\|_{L^{\infty}\left([0, T], H^{m}\right)}<\infty$ uniformly as $\alpha \rightarrow 0$. Suppose we the claim holds for $0 \leq i \leq j-1$. We must prove it for $i=j \leq M$. If $j \geq 1, v_{j}$ and $w_{j}$ satisfy

$$
\begin{aligned}
\left(v_{j}\right)_{t t} & =\left(v_{j}\right)_{x x}+\alpha\left(v_{j}\right)_{x x x x}+N_{j}\left(v_{0}, \ldots, v_{j-1}\right) \\
(1-\alpha \Delta)\left(w_{j}\right)_{t t} & =\left(w_{j}\right)_{x x}+N_{j}\left(w_{0}, \ldots, w_{j-1}\right) .
\end{aligned}
$$

Setting

these can be written as

$$
z_{j}=\left(\begin{array}{c}
v_{j} \\
\left(v_{j}\right)_{t}
\end{array}\right), \quad y_{j}=\left(\begin{array}{c}
w_{j} \\
\left(w_{j}\right)_{t}
\end{array}\right)
$$

$$
\begin{aligned}
\frac{d}{d t} z_{j} & =A z_{j}+\hat{N}_{j}\left(v_{0}, \ldots, v_{j-1}\right), \\
\frac{d}{d t} y_{j} & =B y_{j}+(1-\alpha \Delta)^{-1} \hat{N}_{j}\left(w_{0}, \ldots, w_{j-1}\right),
\end{aligned}
$$

with $\hat{N}_{j}=\left(0, N_{j}\right)^{T}$. Letting

$$
\tau=\left\|\hat{N}_{j}\left(v_{0}, \ldots, v_{j-1}\right)-(1-\alpha \Delta)^{-1} \hat{N}_{j}\left(w_{0}, \ldots, w_{j-1}\right)\right\|_{H^{m} \times H^{m}},
$$

we obtain

$$
\frac{d}{d t}\left\|z_{j}-y_{j}\right\|_{H^{m} \times H^{m}} \leq N^{2}\left(1+\alpha N^{2}\right)\left\|z_{j}-y_{j}\right\|_{H^{m} \times H^{m}}+C(N) \alpha^{2}\left\|y_{j}\right\|_{H^{m}}+\tau .
$$


By (8), for $\alpha$ sufficiently small,

$$
\begin{aligned}
\frac{\tau}{N^{2}} \leq & \sum_{k=0}^{j-1}\left\|v_{k}\right\|_{H^{m}}\left\|v_{j-k-1}-w_{j-k-1}\right\|_{H^{m}}+\sum_{k=0}^{j-1}\left\|w_{j-k-1}\right\|_{H^{m}}\left\|v_{k}-w_{k}\right\|_{H^{m}} \\
& +C \alpha\left\|N_{j}\left(w_{0}, \ldots, w_{j-1}\right)\right\|_{H^{m}} .
\end{aligned}
$$

By the induction hypothesis, there exists a constant $C_{j}(N)$ such that

$$
\sup _{0 \leq t \leq T} \tau \leq C_{j}(N) \alpha
$$

Thus, applying Gronwall's inequality, for $0 \leq t \leq T$,

$$
\left\|z_{j}-y_{j}\right\|_{H^{m} \times H^{m}} \leq\left[C(N) \alpha^{2}\left\|y_{j}\right\|_{L^{\infty}\left([0, t], H^{m}\right)}+C_{j}(N) \alpha\right] e^{N^{2}\left(1+\alpha N^{2}\right) t} .
$$

Observe again that $y_{j}$ is a solution of a linear (finite-dimensional) ordinary differential equation with constant coefficients, and thus again it is bounded for all times, and $\left\|y_{j}\right\|_{L^{\infty}\left([0, T], H^{m}\right)}<\infty$, uniformly as $\alpha \rightarrow 0$. Hence, taking $T \ll 1 / \alpha$, we obtain

$$
\sup _{0 \leq t \leq T}\left\|z_{j}-y_{j}\right\|_{H^{m} \times H^{m}} \leq K_{j}(N) \alpha .
$$

This completes the proof.

\section{Stability of the approximations}

We now establish the stability of the finite-dimensional approximations. That is, we prove that the solutions of (2) and (1) stay close to their respective approximations given in (6)-(7) and (9)-(10). This is respectively the content of Lemmas 4 and 5.

Lemma 4. Fix $T>0, C>0$ and $\delta>0$. Choose $\epsilon$ sufficiently small so that $T<T_{\sharp}$. Assume that $\alpha$ is sufficiently small, and $N$, the number of nonzero Fourier modes in the finite dimensional approximations, is sufficiently large. Let $u$ be any solution of (2) with initial conditions in $H^{m+2} \times H^{m}$ on the central manifold and with norm bounded by $C$. Then

$$
\tilde{u}=v_{0}+\varepsilon v_{1}+\cdots+\varepsilon^{M} v_{M}
$$

satisfies

$$
\|u-\tilde{u}\|_{L^{\infty}\left([0, T], H^{m} \times H^{m-2}\right)}<\delta .
$$

We note that the bounds in the lemma depend only on the initial conditions for $u$ and $\tilde{u}$ in $H^{m+2} \times H^{m}$ and not on its difference. If we assume the norm in $H^{m+2} \times H^{m}$ to be $O\left(\epsilon^{k}\right)$ then one can take the time polinomially large in $\frac{1}{\epsilon}$. However, we do not want to make this additional assumption although we indicate in the proof how it could be used. Another remark is that the fact that $T$ is bounded by $T_{\sharp}$ is not restrictive since it blows up as $\epsilon \rightarrow 0$, which is the limit we will use. 
Proof. Define for $m \geq 2$ the quantity

$$
E_{1}=\|u-\tilde{u}\|_{H^{m}}^{2}+\left\|u_{t}-\tilde{u}_{t}\right\|_{H^{m-2}}^{2} .
$$

If we choose $N$ large enough we can make $E_{1}(0)$ arbitrarily small.

We claim that there exist constants $K_{1}$ and $K_{2}$ such that

$$
\frac{d}{d t} E_{1} \leq K_{1} E_{1}^{1 / 2}+K_{2} \varepsilon^{M+2}
$$

If we establish this estimate the lemma will follow. The constants $K_{1}$ and $K_{2}$ depend on bounds for the solution $u$ and the approximated solution $\tilde{u}$ in $H^{m+2} \times H^{m}$, that can be estimated from the initial data. Under additional smallness conditions for the initial data, as in the remark after the statement of the lemma, one may improve the results by showing, for instance, that $K_{1}=K \epsilon^{k}$. Then one could estimate

$$
K_{1} E_{1}^{1 / 2} \leq C \epsilon E_{1}+O\left(\epsilon^{2 k-1}\right)
$$

which would yield estimates valid up to $T=\frac{1}{\epsilon}$.

To prove (12) let us compute $\frac{d}{d t} E_{1}$. We can rewrite (2) and (6)-(7) as

$$
\frac{d}{d t} z_{1}=A z_{1}+\varepsilon M_{1}\left(z_{1}\right) \text { and } \frac{d}{d t} z_{2}=A z_{2}+\varepsilon M_{2}\left(z_{2}\right),
$$

with $A$ as in (11), and with

$$
z_{1}=\left(\begin{array}{c}
u \\
u_{t}
\end{array}\right), \quad z_{2}=\left(\begin{array}{c}
\tilde{u} \\
\tilde{u}_{t}
\end{array}\right), \quad M_{1}\left(z_{1}\right)=\left(\begin{array}{c}
0 \\
\left(u^{2}\right)_{x x}
\end{array}\right)
$$

and

$$
M_{2}\left(z_{2}\right)=\left(\left(\tilde{u}^{2}-\sum_{i+j \geq M}^{0} \varepsilon^{i+j} v_{i} v_{j}\right)_{x x}\right) .
$$

Note that $z_{i} \in H^{m+2} \times H^{m}, i=1,2$. For $z_{1}$ this holds by the energy estimates and the assumption on the initial data. For $z_{2}$ this is an immediate consequence of the fact that there are only finitely many Fourier modes different from zero - therefore $z_{2}$ is bounded in any Sobolev space.

Moreover, if we define

$$
\mathcal{I}=\frac{d}{d t}\|u-\tilde{u}\|_{H^{m}}^{2}, \quad \mathcal{J}=\frac{d}{d t}\left\|u_{t}-\tilde{u}_{t}\right\|_{H^{m-2}}^{2},
$$

then $\frac{d}{d t} E_{1}=\mathcal{I}+\mathcal{J}$. We have

$$
\mathcal{I}=2 \sum_{0<n \leq m} \int_{0}^{1}\left[D^{n}(u-\tilde{u})\right]\left[D^{n}\left(u_{t}-\tilde{u}_{t}\right)\right] d x .
$$

Integrating by parts,

$$
\mathcal{I}=-2 \sum_{0<n \leq m} \int_{0}^{1}\left[D^{n+1}(u-\tilde{u})\right]\left[D^{n-1}\left(u_{t}-\tilde{u}_{t}\right)\right] d x,
$$


and using Young's inequality,

$$
\begin{aligned}
|\mathcal{I}| & \leq \sum_{0<n \leq m}\left[\int_{0}^{1}\left|D^{n+1}(u-\tilde{u})\right|^{2} d x+\int_{0}^{1}\left|D^{n-1}\left(u_{t}-\tilde{u}_{t}\right)\right|^{2} d x\right] \\
& =\|u-\tilde{u}\|_{H^{m+1}}^{2}+\left\|u_{t}-\tilde{u}_{t}\right\|_{H^{m-1}}^{2} .
\end{aligned}
$$

By itself, this estimate is not sufficient to control the norm of the solutions since the energy $E_{1}$ does not control the $H^{m+1}$ norms of $u-\tilde{u}$ or the $H^{m-1}$ norms of $u_{t}-\tilde{u}_{t}$. However, we know that these norms are bounded a priori in $H^{m+2}$ and $H^{m}$, although they may not be small in these spaces. Therefore, by interpolation inequalities, there exist constants $C_{1}$ and $C_{2}$ such that

$$
|\mathcal{I}| \leq C_{1}\|u-\tilde{u}\|_{H^{m}}\|u-\tilde{u}\|_{H^{m+2}}+C_{2}\left\|u_{t}-\tilde{u}_{t}\right\|_{H^{m}}\left\|u_{t}-\tilde{u}_{t}\right\|_{H^{m-2}} .
$$

Recall that $u, \tilde{u} \in H^{m+2}$ and $u_{t}, \tilde{u}_{t} \in H^{m}$. Define

$$
\hat{C}_{1}=C_{1}\|u-\tilde{u}\|_{L^{\infty}\left([0, T], H^{m+2}\right)}, \quad \hat{C}_{2}=C_{2}\left\|u_{t}-\tilde{u}_{t}\right\|_{L^{\infty}\left([0, T], H^{m}\right)} .
$$

We conclude that

$$
|\mathcal{I}| \leq \max \left\{\hat{C}_{1}, \hat{C}_{2}\right\}\left(\|u-\tilde{u}\|_{H^{m}}+\left\|u_{t}-\tilde{u}_{t}\right\|_{H^{m-2}}\right) \leq \tilde{C}_{1} E_{1}^{1 / 2} .
$$

Since

$$
\|u-\tilde{u}\|_{H^{m+2}} \leq\|u\|_{H^{m+2}}+\|\tilde{u}\|_{H^{m+2}},
$$

and

$$
\left\|u_{t}-\tilde{u}_{t}\right\|_{H^{m}} \leq\left\|u_{t}\right\|_{H^{m}}+\left\|\tilde{u}_{t}\right\|_{H^{m}},
$$

$\hat{C}_{1}$ and $\hat{C}_{2}$ can be estimated from the initial conditions. The main point here is not that $\|u-\tilde{u}\|_{L^{\infty}\left([0, T], H^{m+2}\right)}$ and $\left\|u_{t}-\tilde{u}_{t}\right\|_{L^{\infty}\left([0, T], H^{m}\right)}$ are small, but that they are bounded by the initial data.

We now bound $\mathcal{J}$. Let us denote

$$
V=\sum_{i+j \geq M} \varepsilon^{i+j-M} v_{i} v_{j}
$$

In an analogous manner to that with $\mathcal{I}$, we have

$$
\begin{aligned}
& \mathcal{J}=2 \sum_{0<n \leq m-2} \int_{0}^{1}\left[D^{n}\left(u_{t}-\tilde{u}_{t}\right)\right]\left(\left[D^{n+2}(u-\tilde{u})\right]+\alpha\left[D^{n+4}(u-\tilde{u})\right]\right) d x \\
& +2 \varepsilon \sum_{0<n \leq m-2} \int_{0}^{1}\left[D^{n}\left(u_{t}-\tilde{u}_{t}\right)\right]\left[D^{n+2}\left(u^{2}-\tilde{u}^{2}\right)\right] d x \\
& +2 \varepsilon^{M+1} \sum_{0<n \leq m-2} \int_{0}^{1}\left[D^{n}\left(u_{t}-\tilde{u}_{t}\right)\right]\left[D^{n+2} V\right] d x .
\end{aligned}
$$


Integrating by parts,

$$
\begin{aligned}
& \mathcal{J}=-2 \sum_{0<n \leq m-2} \int_{0}^{1}\left[D^{n+1}\left(u_{t}-\tilde{u}_{t}\right)\right]\left[D^{n+1}(u-\tilde{u})\right] d x \\
& -2 \sum_{0<n \leq m-2} \int_{0}^{1}\left[D^{n+1}\left(u_{t}-\tilde{u}_{t}\right)\right]\left(\alpha\left[D^{n+3}(u-\tilde{u})\right]+\varepsilon\left[D^{n+1}\left(u^{2}-\tilde{u}^{2}\right)\right]\right) d x \\
& +2 \varepsilon^{M+1} \sum_{0<n \leq m-2} \int_{0}^{1}\left[D^{n}\left(u_{t}-\tilde{u}_{t}\right)\right]\left[D^{n+2} V\right] d x .
\end{aligned}
$$

Using Young's inequality, we obtain

$$
\begin{aligned}
& |\mathcal{J}| \leq\left(1+\alpha+\varepsilon+2 \varepsilon^{M+2}\right)\left\|u_{t}-\tilde{u}_{t}\right\|_{H^{m-1}}^{2}+(1+\alpha)\|u-\tilde{u}\|_{H^{m+1}}^{2} \\
& +\varepsilon C\|u-\tilde{u}\|_{H^{m-1}}^{2}\|u+\tilde{u}\|_{H^{m-1}}^{2}+C \varepsilon^{M+1}\|V\|_{H^{m}}^{2},
\end{aligned}
$$

in which we used the fact that $H^{m}, m \geq 1 / 2$ is an algebra.

By interpolation inequalities, there exist constants $C_{3}, C_{4}, C_{5}$, and $K_{2}$ such that

$$
\begin{aligned}
|\mathcal{J}| & \leq C_{3}\left(1+\alpha+\varepsilon+2 \varepsilon^{M+2}\right)\left\|u_{t}-\tilde{u}_{t}\right\|_{H^{m-2}}\left\|u_{t}-\tilde{u}_{t}\right\|_{H^{m}}+ \\
& +C_{4}(1+\alpha)\|u-\tilde{u}\|_{H^{m}}\|u-\tilde{u}\|_{H^{m+2}}+C_{5} \varepsilon\|u-\tilde{u}\|_{H^{m}}+K_{2} \varepsilon^{M+1}
\end{aligned}
$$

in which

$$
C_{5}=\|u-\tilde{u}\|_{H^{m-1}}\|u+\tilde{u}\|_{H^{m-1}}^{2}
$$

and $K_{2}=\|V\|_{H^{m}}^{2}$. Since $u-\tilde{u} \in H^{m+2}, u_{t}-\tilde{u}_{t} \in H^{m}$, defining

$$
\hat{C}_{3}=C_{3}\left(1+\alpha+\varepsilon+2 \varepsilon^{M+2}\right)\left\|u_{z}-\tilde{u}_{t}\right\|_{L^{\infty}\left([0, T], H^{m}\right)},
$$

and

we obtain

$$
\hat{C}_{4}=C_{4}(1+\alpha)\|u-\tilde{u}\|_{L^{\infty}\left([0, T], H^{m+2}\right)}+C_{5} \varepsilon
$$

$$
|\mathcal{J}| \leq \tilde{C}_{2} E_{1}^{1 / 2}+K_{2} \varepsilon^{M+2}
$$

where $\tilde{C}_{2} \leq 2 \max \left\{\hat{C}_{3}, \hat{C}_{4}\right\}$. Thus,

$$
\frac{d}{d t} E_{1} \leq|\mathcal{I}|+|\mathcal{J}| \leq K_{1} E_{1}^{1 / 2}+K_{2} \varepsilon^{M+2} .
$$

This implies the desired result.

Lemma 5. Fix $T>0, C>0$ and $\delta>0$. Choose $\epsilon$ and $\frac{\epsilon}{\alpha^{1 / 2}}$ sufficiently small so that $T<T_{*}$. Assume that $\alpha$ is sufficiently small and $N$, the number of nonzero Fourier modes in the finite dimensional approximations, is sufficiently large. Let $u$ be any solution of (1) with initial conditions in $H^{m} \times H^{m}$ with norm bounded by $C$. Then the approximation

$$
\bar{u}=w_{0}+\varepsilon w_{1}+\cdots+\varepsilon^{M} w_{M},
$$

satisfies

$$
\|u-\bar{u}\|_{L^{i} n f t y\left([0, T], H^{m} \times H^{m}\right)}<\delta .
$$


As in the previous lemma these estimates can hold for polynomially large times in $\frac{1}{\epsilon}$ if one assumes further estimates on the norm of $u$ in $H^{m}$.

Proof. The function $z=u-\bar{u}$ satisfies

$$
(1-\alpha \Delta) z_{t t}=z_{x x}+\varepsilon\left(u^{2}-\bar{u}^{2}\right)_{x x}+\varepsilon^{M+1}\left(\sum_{i+j \geq M} \varepsilon^{i+j-M} w_{i} w_{j}\right)_{x x} .
$$

Define

$$
W=\sum_{i+j \geq M} \varepsilon^{i+j-M} w_{i} w_{j}
$$

We apply $D^{m-1}$ to (13), and multiply it by $D^{m-1} z_{t}$ to get

$$
\begin{aligned}
& \left(D^{m-1}(1-\alpha \Delta) z_{t t}\right)\left(D^{m-1} z_{t}\right)=\left(D^{m-1} z_{x x}\right)\left(D^{m-1} z_{t}\right) \\
& +\varepsilon D^{m+1}\left[u^{2}-\bar{u}^{2}\right]\left(D^{m-1} z_{t}\right)+\varepsilon^{M+1}\left(D^{m+1} W\right)\left(D^{m-1} z_{t}\right) .
\end{aligned}
$$

Integrating by parts, we can write (14) in the form

$$
\begin{aligned}
& \frac{1}{2} \frac{d}{d t} \int_{0}^{1}\left(\left(D^{m-1} z_{t}\right)^{2}+\alpha\left(D^{m} z_{t}\right)^{2}+\left(D^{m} z\right)^{2}\right) d x= \\
& =-\varepsilon \int_{0}^{1}\left(D^{m} z_{t}\right)\left[D^{m}\left(u^{2}-\bar{u}^{2}\right)\right] d x+\varepsilon^{M+1} \int_{0}^{1}\left(D^{m+1} W\right)\left(D^{m-1} z_{t}\right) d x
\end{aligned}
$$

Setting

$$
E=\int_{0}^{1}\left(\left(D^{m-1} z_{t}\right)^{2}+\alpha\left(D^{m} z_{t}\right)^{2}+\left(D^{m} z\right)^{2}\right) d x
$$

using the Cauchy-Schwartz inequality, and the bounds

$$
\int_{0}^{1}\left(D^{m} z_{t}\right)^{2} d x \leq \frac{E}{\alpha}, \quad \int_{0}^{1}\left(D^{m-1} z_{t}\right)^{2} d x \leq E,
$$

we conclude that

$$
\frac{1}{2} \frac{d}{d t} E \leq \frac{\varepsilon E^{1 / 2}}{\alpha^{1 / 2}}\left\|u^{2}-\bar{u}^{2}\right\|_{H^{m}}+\varepsilon^{M+1} E^{1 / 2}\|W\|_{H^{m+1}} .
$$

Since $H^{m}, m>1 / 2$ is an algebra,

$$
\frac{1}{2} \frac{d}{d t} E \leq \frac{C \varepsilon}{\alpha^{1 / 2}} E^{1 / 2}\|z\|_{H^{m}}\|u+\bar{u}\|_{H^{m}}+\varepsilon^{M+1} E^{1 / 2}\|W\|_{H^{m+1}} .
$$

By Poincaré's inequality, $\|z\|_{H^{m}} \leq C E^{1 / 2}$ for some constant $C>0$. Thus,

$$
\frac{d}{d t} E \leq \frac{C \varepsilon}{\alpha^{1 / 2}} E^{3 / 2}+\frac{C \varepsilon}{\alpha^{1 / 2}} E\|u+\bar{u}\|_{H^{m}}+\varepsilon^{M+1} E^{1 / 2}\|W\|_{H^{m+1}} .
$$

Using Young's inequality,

$$
E \leq \frac{2}{3} E^{3 / 2}+\frac{1}{3}, \quad E^{1 / 2} \leq \frac{1}{3} E^{3 / 2}+\frac{2}{3},
$$

and we obtain

$$
\frac{d}{d t} E \leq C_{1} E^{3 / 2}+C_{2},
$$


where

$$
C_{1}=\frac{C \varepsilon}{\alpha^{1 / 2}}\left(1+\frac{2}{3}\|u+\bar{u}\|_{H^{m}}\right)+\frac{\varepsilon^{M+1}}{3}\|W\|_{H^{m+1}}
$$

and

Since

$$
C_{2}=\frac{C \varepsilon}{\alpha^{1 / 2}}\|u+\bar{u}\|_{H^{m}}+\frac{2}{3} \varepsilon^{M+1}\|W\|_{H^{m+1}} .
$$

$$
\frac{d}{d t} E \leq C_{1} E^{3 / 2}+C_{2} \leq\left(C_{1}^{2 / 3} E+C_{2}^{2 / 3}\right)^{3 / 2},
$$

solving the ordinary differential equations in the equality case, we obtain

$$
E(t) \leq \frac{4-C_{1}^{4 / 3} C_{2}^{2 / 3}(t-K)^{2}}{C_{1}^{2}(t-K)^{2}}
$$

with $K=2 /\left[C_{1}^{4 / 3} C_{2}^{2 / 3}+C_{1}^{2} E(0)\right]^{1 / 2}$. This completes the proof.

The main result of this paper is the following.

Theorem 6. Fix $T>0, C>0$ and $\delta>0$. Choose $\epsilon$ and $\frac{\epsilon}{\alpha^{1 / 2}}$ sufficiently small so that $T<T_{\sharp}, T_{*}$. Assume that $\alpha$ is sufficiently small, and $N$, the number of nonzero Fourier modes in the finite dimensional approximations, is sufficiently large. Suppose that $u_{1}$ is a solution of (1) and that $u_{2}$ is a solution of (2) in its center manifold, both with norms bounded by $C$. Assume further that the initial data for the two equations coincides in $H^{m} \times$ $H^{m-2}$ at $t=0$. Then

$$
\left\|u_{1}-u_{2}\right\|_{L^{\infty}\left([0, T], H^{m}\right)}<\delta .
$$

Proof of Theorem 6. First observe that the solution $u_{2}$ in the center manifold is bounded in $H^{m+2} \times H^{m}$ for a time $T$ that blows up as the norm of the initial data goes to zero or $\varepsilon \rightarrow 0$.

If $\alpha$ is sufficiently small, it is possible to approximate the initial conditions of $u_{2}$ arbitrarily well by $\tilde{u}_{2}$ by truncating it to finitely many Fourier modes. Then, since $u_{2}$ is itself bounded, the constant $K_{1}$ in Lemma 4 is bounded. Therefore, by Lemma 4 , for $\varepsilon$ sufficiently small, $u_{2}$ and $\tilde{u}_{2}$ stay close. The approximations $\tilde{u}_{2}$ and $\bar{u}_{1}$ stay close together as long as $\alpha$ is sufficiently small, by Lemma 3. By Theorem 1 the solution $u_{1}$ exists, and is bounded, for times that blow up as $\varepsilon / \alpha^{1 / 2} \rightarrow 0$. Finally, Lemma 5 says that $\bar{u}_{1}$ and $u_{1}$ stay close together, as long as $\varepsilon / \alpha^{1 / 2}$ and $\varepsilon$ are sufficiently small.

\section{REFERENCES}

[1] J. L. Bona et al., Global existence of smooth solutions and stability of solitary waves for a generalized Boussinesq equation, Comm. Math. Phys. 118 (1988), no. 1, 15-29.

[2] J. Boussinesq, Theorie des ondes et des remous qui se propagent le long d'un canal rectangular horizontal, J. Math. Pure Appl. 7 (1872), 55-108.

[3] W. Craig, An existence theory for water waves and the Boussinesq and Korteweg-de Vries scaling limits, Comm. Partial Differential Equations 10 (1985), 787-1003.

[4] R. de la Llave and C. Valls, A smooth center manifold theorem for ill posed differential equations in Banach spaces, in preparation. 
[5] T. Kano and T. Nishida, Sur l'équation de Boussinesq des ondes de surface de l'eau, Proc. Japan Acad. Ser. A Math. Sci. 61 (1985), 91-94.

[6] A. Mielke, Hamiltonian and Lagrangian flows on center manifolds, Lecture Notes in Mathematics, 1489, Springer-Verlag, Berlin, 1991.

[7] J. Shatah and M. Struwe, Geometric wave equations, New York University, Courant Institute of Mathematical Sciences, New York, 1998.

Departamento de Matemática, Instituto Superior TÉcnico, 1049-001 Lisboa, PorTugal

E-mail address: dgomes@math.ist.utl.pt

Departamento de Matemática, Instituto Superior Técnico, 1049-001 Lisboa, PorTugal

E-mail address: cvalls@math.ist.utl.pt 